\title{
Experimental and computational analyses of electroabsorption in polar InGaN/GaN quantum zigzag heterostructures
}

\author{
Emre Sari ${ }^{1}$, Tuncay Ozel ${ }^{1}$, Asli Koc ${ }^{1}$, Jin-Woo Ju ${ }^{2}$, Haeng-Keun Ahn' ${ }^{2}$ In-Hwan Lee ${ }^{2}$, \\ Jong Hyeob Baek ${ }^{3}$, and Hilmi Volkan Demir ${ }^{1 *}$ \\ ${ }^{1}$ Department of Electrical and Electronics Engineering, Department of Physics, Nanotechnology Research Center, \\ Institute of Materials Science and Nanotechnology, Bilkent University, Bilkent, Ankara 06800 Turkey \\ ${ }^{2}$ School of Advanced Materials Engineering, Research Center of Industrial Technology, Chonbuk National University, Chonju 561-756, Korea \\ ${ }^{3}$ Korea Photonics Technology Institute, Gwangju 500-460, Korea \\ *Tel:[+90](312) 290 1021, e-mail: volkan@bilkent.edu.tr
}

Traditional quantum confined Stark effect is well known to lead to strong electroabsorption in multiple quantum well (MQW) structures, yielding only red-shift of the absorption edge with the externally applied electric field, independent of the direction of the applied field [1]. However, a little is known the electroabsorption behavior in IIInitride quantum structures grown on c-plane of their wurtzite crystal structure, which is substantially different than the electroabsorption of conventional quantum structures. Such III-N heterostructures exhibit strong polarization fields and discontinuity of such polarization fields at their heterointerfaces causes stimulation of large electrostatic fields in alternating directions for their wells and barriers [2]. Consequently, their energy band diagrams form a zigzag potential profile in conduction and valence bands, instead of those with square profiles. A natural and suitable approach for understanding these polarization fields and also developing insight to design related devices (e.g., electroabsorption modulators) is to study electroabsorption behavior as a function of the polarization field in such polar structures $[3,4]$. To this end, we present a comparative, computational and experimental study of electroabsorption in our different designs of c-plane grown polar $\mathrm{InGaN} / \mathrm{GaN}$ quantum structures with varying levels of polarization.

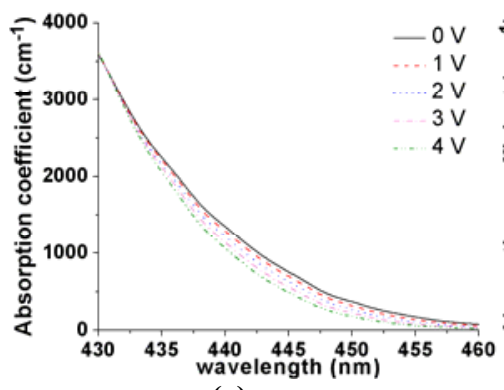

(a)

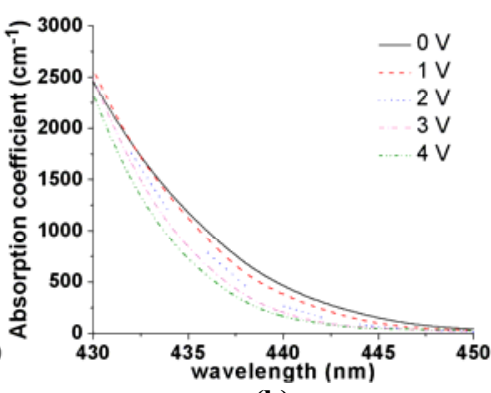

(b)

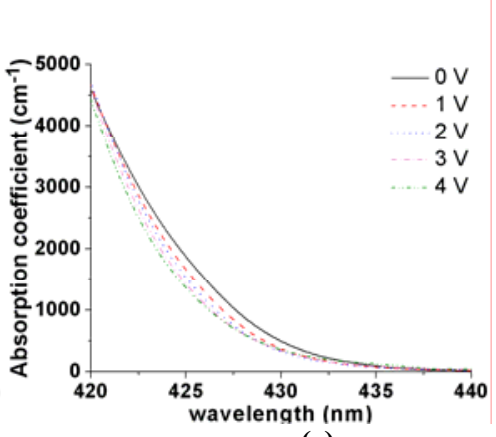

(c)

Figure 1 Spectral absorption tails of Samples A, B, and C under reverse bias of 1-4 V in (a), (b), and (c), respectively.

For a comparative study, we employed p-i-n diode architecture that incorporated InGaN/GaN quantum structures [3, 4]. Among three sets of samples (Sample A, B, and C) used in this work, structural parameters of the active MQW regions were changed. These structural parameters included $\mathrm{InN}$ concentrations in the quantum well regions and well-to-barrier-width ratios. By controlling these two parameters, we varied the level of polarization induced electrostatic fields to investigate its effect on electroabsorption. The active region of the three samples included quantum well/quantum barrier structures with $2.5 \mathrm{~nm} / 7.5 \mathrm{~nm} \operatorname{In}_{0.15} \mathrm{Ga}_{0.85} \mathrm{~N} / \mathrm{GaN}$ in Sample A, $3 \mathrm{~nm} / 4 \mathrm{~nm}$ $\mathrm{In}_{0.15} \mathrm{Ga}_{0.85} \mathrm{~N} / \mathrm{GaN}$ in Sample B, and $3 \mathrm{~nm} / 4 \mathrm{~nm} \operatorname{In}_{0.12} \mathrm{Ga}_{0.88} \mathrm{~N} / \mathrm{GaN}$ in Sample C. For each sample, we computed the built-in polarization-induced electric fields across the wells: $-383 \mathrm{~V} / \mu \mathrm{m}$ for Sample A, $-300 \mathrm{~V} / \mu \mathrm{m}$ for Sample B, and $-238 \mathrm{~V} / \mu \mathrm{m}$ for Sample C. Those across the barriers were calculated to be $127 \mathrm{~V} / \mu \mathrm{m}$ for Sample A, $220 \mathrm{~V} / \mu \mathrm{m}$ for Sample B, and $179 \mathrm{~V} / \mu \mathrm{m}$ for Sample C. Using transfer matrix method under effective mass approximation, we computed electron and hole eigenstate wavefunctions and their energy eigenvalues for each sample under different levels of externally applied electric fields. From the overlap integral of the resulting electron and hole wavefunctions, we deduced the predicted relative absorption coefficient changes with the ratios of 0.4 around $\lambda=450$ $\mathrm{nm}$ for Sample A, 0.53 around $\lambda=440 \mathrm{~nm}$ for Sample B, and 1.0 around $\lambda=420 \mathrm{~nm}$ for Sample C, all for a field swing of $40 \mathrm{~V} / \mu \mathrm{m}$. From our computational analysis, we predicted the strongest electroabsorption behavior in Sample C with the weakest polarization-induced electrostatic field inside its quantum wells.

For experimental implementation, using metal organic chemical vapor deposition (MOCVD), we grew our p-i-n diodes that were verified to embed these designed quantum structures by double crystal X-ray diffraction (DCXRD) $[3,4]$. Subsequently, following our standard fabrication procedures to make contacts and device mesas, we 
performed electroabsorption measurements (Fig. 1) on these fabricated epi-devices, using a broadband Xenon lamp, a monochromator, a chopper, a DC voltage source, and a lock-in amplifier. The absorption coefficient change measurements (Fig. 2) revealed a maximum absorption coefficient change of $288 \mathrm{~cm}^{-1}$ at $\lambda=440 \mathrm{~nm}$ for Sample A, $462 \mathrm{~cm}^{-1}$ at $\lambda=434 \mathrm{~nm}$ for Sample B, and $555 \mathrm{~cm}^{-1}$ at $\lambda=422 \mathrm{~nm}$ for Sample C, all of which were obtained under a $0-4 \mathrm{~V}$ swing, corresponding to a field change of $40 \mathrm{~V} / \mu \mathrm{m}$. These led to the relative absorption coefficient changes with the ratios of 0.52 at $\lambda=440 \mathrm{~nm}$ for Sample A, 0.83 at $\lambda=434 \mathrm{~nm}$ for Sample B, and 1.0 at $\lambda=422 \mathrm{~nm}$ for Sample $\mathrm{C}$ with the same electric field swing. As a result, our experimental demonstrated that electroabsorption in these polar heterostructures grew stronger with weaker polarization-induced electrostatic fields inside their quantum wells, also verifying our computational results.

In our computational and experimental analyses, unlike conventional QCSE, we observed that these III-N polar MQWs exhibited blue-shifting electroabsorption, when the external electric field was applied to compensate for the polarization-induced electric field across their wells. However, the same InGaN/GaN quantum structures red-shifted their absorption edge when the direction of the external field was reversed (so that the external field was applied in the same direction as the well polarization), as shown in the direction-dependent electroabsorption behavior under 0 , +1 and $-1 \mathrm{~V}$ external biases (Fig. 3). In these polar structures, polarization broke symmetry and direction of the applied field made a difference. Such co-observation of blue- and red-shifts in the electroabsorption of polar III-N quantum heterostructures is a unique property, which is impossible in non-polar quantum heterostructures,

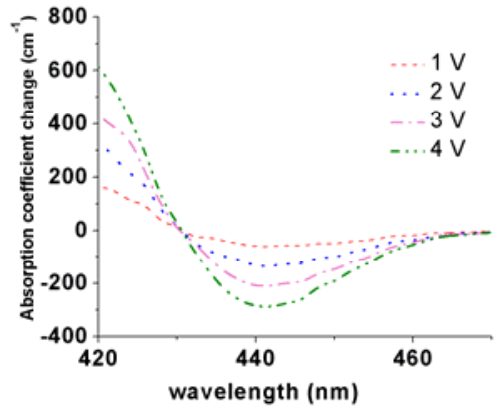

(a)

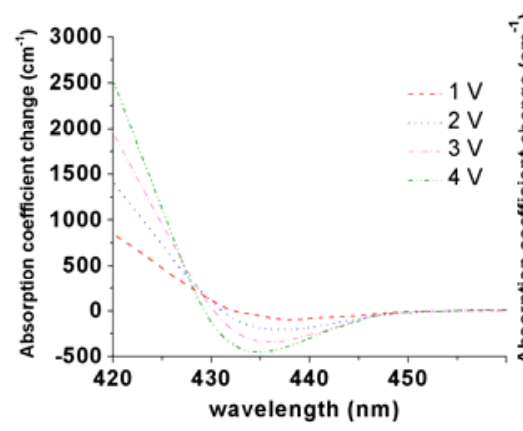

(b)

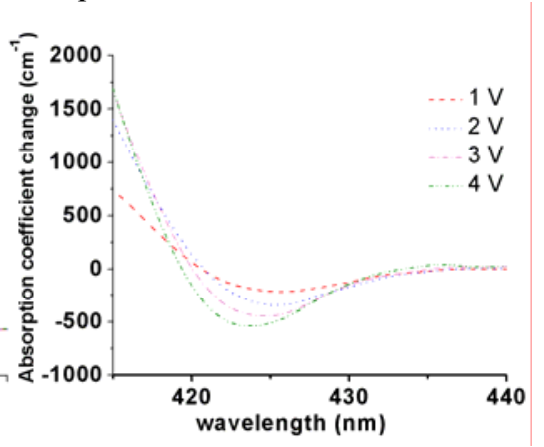

(c)

Figure 2 Spectral absorption coefficient changes in Samples A, B, and C under reverse bias of 1-4 V with respect to no bias case in (a), (b), and (c), respectively.

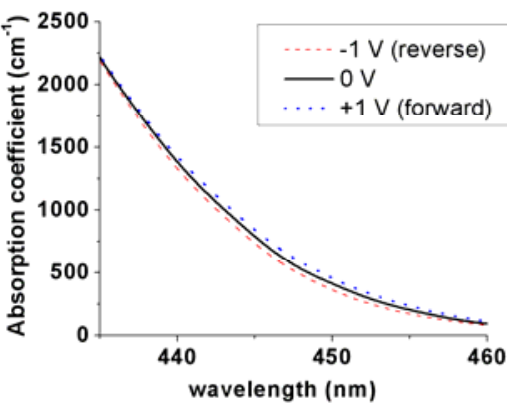

(a)

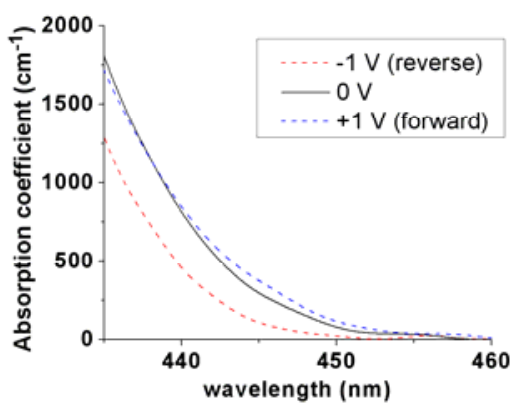

(b)

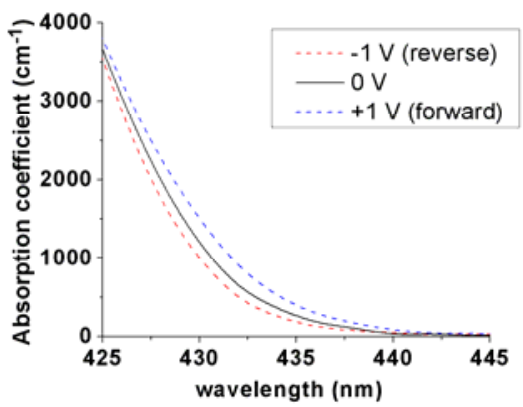

(c)

Figure 3 Absorption spectra of Samples A, B, and C given in (a), (b), and (c), respectively, under biases of -1V, 0V, and 1V.

In conclusion, we investigated electroabsorption in polar InGaN/GaN quantum structures both theoretically and experimentally. In the same polar quantum structures, we showed both blue- and red-shifting absorption edges depending on the direction of the applied field. We also demonstrated that electroabsorption grew stronger with reduced polarization-induced electrostatic fields.

Acknowledgements: This work was supported by EU MOON, EU NOE PHOREMOST and TUBITAK 104E114, 107E297, 106E020, 107E088, 105E065, and 105E066. H.V.D. and E.S. also acknowledge additional support from ESF-EURYI, TUBA-GEBIP and TUBITAK.

References

[1] D.A.B. Miller, et al., Phys. Rev. B, 32, 1043 (1985).

[2] V. Fiorentini, et al., Phys. Rev. B, 60, 8849 (1999).

[3] E. Sari, et al., Appl. Phys. Lett., 90, 011101 (2007).

[4] E. Sari, et al., Appl. Phys. Lett., 92, 201105 (2008). 